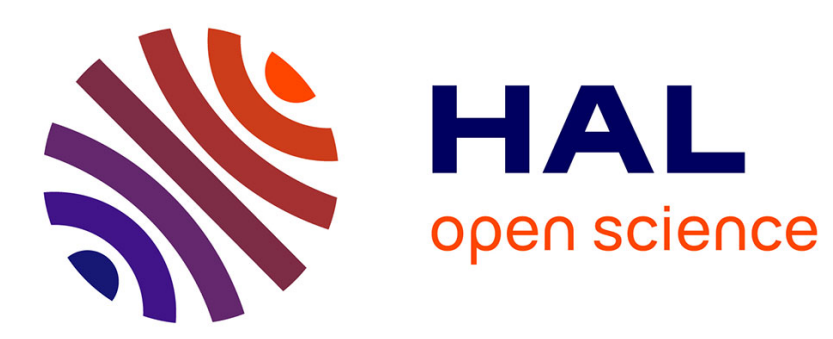

\title{
Les bases de la génétique quantitative. Le progrès génétique et sa réalisation dans les expériences de sélection
}

Hubert de Rochambeau

\section{To cite this version:}

Hubert de Rochambeau. Les bases de la génétique quantitative. Le progrès génétique et sa réalisation dans les expériences de sélection. Productions Animales, 1992, 1992, pp.83-86. hal-02701319

\section{HAL Id: hal-02701319 \\ https://hal.inrae.fr/hal-02701319}

Submitted on 1 Jun 2020

HAL is a multi-disciplinary open access archive for the deposit and dissemination of scientific research documents, whether they are published or not. The documents may come from teaching and research institutions in France or abroad, or from public or private research centers.
L'archive ouverte pluridisciplinaire HAL, est destinée au dépôt et à la diffusion de documents scientifiques de niveau recherche, publiés ou non, émanant des établissements d'enseignement et de recherche français ou étrangers, des laboratoires publics ou privés. 


\section{H. DE ROCHAMBEAU}

INRA Station d'Amélioration Génétique des Animaux BP27 31326 Castanet-Tolosan Cedex

\section{Les bases de la génétique quantitative}

\section{Le progrès génétique et sa réalisation dans les expériences de sélection}

Résumé. La génétique quantitative fournit un modèle pour prévoir a priori le progrès génétique. Il est fonction de l'intensité de sélection, de la précision, de la racine carrée de la variance génétique additive et de l'intervalle entre générations. Liintensité de sélection est fonction de la différentielle de sélection ou du taux de sélection. La précision dépend de l'héritabilité du caractère sélectionné et de la méthode utilisée. L'intervalle entre générations est l'âge moyen des parents à la naissance de leurs descendants qui seront soumis à la sélection pour produire la génération suivante.

La réalisation d'expériences de sélection confirme la validité de ce modèle, tout en montrant ses limites. Dans le cas d'une expérience de sélection sur le gain de poids post-sevrage chez la souris on observe, après 14 générations, une réponse qui augmente avec lintensité de sélection et avec la taille de la population. Dans le cas d'une expérience de sélection pour le nombre d'oeufs chez la poule, la sélection est toujours efficace après 30 générations.

Il existe différentes modalités de sélection. La sélection individuelle ou massale consiste à choisir les individus d'après leurs propres performances. Les performances des individus apparentés aux candidats sont des sources d'information complémentaires. On distingue classiquement trois types d'apparentés (les ascendants, les collatéraux et les descendants), et donc trois autres modalités : la sélection sur ascendance, sur collatéraux et sur descendance.

La sélection artificielle consiste à choisir des mâles et des femelles dans une population et à les faire reproduire en espérant que les descendants seront meilleurs. Cette pratique est aussi vieille que l'élevage. La génétique quantitative fournit un modèle pour prévoir a priori le gain génétique et le progrès génétique. La réalisation d'expériences de sélection confirme la validité de ce modèle, tout en montrant ses limites.

\section{1 / Expression du progrès génétique}

On calcule le gain génétique $R$ pour un caractère, où le phénotype $\mathrm{P}$ s'écrit $\mathrm{P}=\mathrm{A}+\mathrm{E}$. Il n'y a pas de dominance et pas d'épistasie. Les moyennes des performances et des valeurs génétiques additives dans cette grande population sont identiques : $\mathrm{P}=\mathrm{A}$ car $\mathrm{E}=0$.

La sélection, dite massale, se fait par troncature dans une distribution normale : un seuil fixé - $\mathrm{P}_{\mathrm{t}}$ sépare la population en deux groupes, les reproducteurs sélectionnés et les animaux éliminés de la reproduction. On choisit les reproducteurs d'après leur propre performance. Les accouplements, entre les reproducteurs sélectionnés, se font au hasard (panmixie) et les générations sont séparées.

\section{1 / Le gain génétique $R$}

La figure 1 décrit un cycle de sélection dans lequel l'effort d'amélioration porte sur un caractère. La sélection a pour but d'améliorer la valeur additive moyenne A en sélectionnant les candidats selon leurs propres performances $P$. On retient comme reproducteurs les individus dont la performance est supérieure à $\mathrm{P}_{\mathrm{t}}$ (partie hachurée de la figure 1). Leur performance moyenne est $\mathrm{P}_{\mathrm{s}}$. La quantité $\mathrm{S}=\mathrm{P}_{\mathrm{s}}-\mathrm{P}_{0}$ est appelée différentielle de sélection. C'est une mesure de la pression de sélection appliquée. La distribution des valeurs additives des individus sélectionnés, ainsi que leur valeur moyenne sont inconnues. On sait cependant que leur valeur additive moyenne est inférieure à leur performance moyenne. Les individus sélectionnés ont bénéficié d'effets positifs aléatoires de l'environnement. La distribution des valeurs génétiques $\mathrm{A}$ des individus sélectionnés est donc décalée sur la gauche par rapport à la distribution de leurs performances. $P_{1}$ est la valeur additive moyenne du groupe des reproducteurs sélectionnés. Ce groupe se reproduit en panmixie. La population des descendants a une performance moyenne $P_{1}$, qui est aussi sa valeur additive moyenne. La différence $R=P_{1}-P_{0}$ est la réponse à la sélection ou le gain génétique. La réponse serait égale à la différentielle si l'environnement n'était plus une source de variation. Le rapport $\mathrm{R} / \mathrm{S}$ est l'augmentation de la valeur additive $\mathrm{A}$ par unité d'augmentation du critère de sélection $P$; c'est- 
Figure 1.

Description

d'un cycle de

sélection

(d'après

Minvielle)

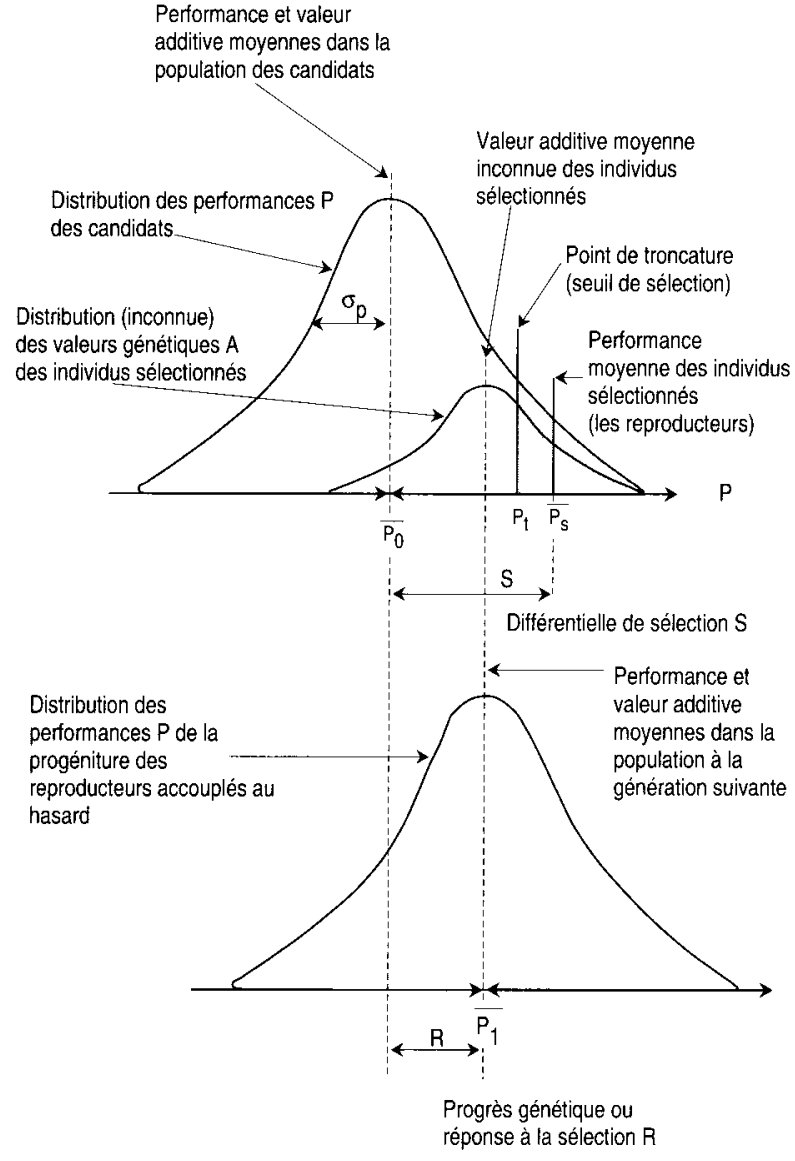

à-dire le coefficient de régression $\mathrm{b}_{\mathrm{AP}}$ de la valeur additive sur la performance.

$\mathrm{R}=\mathrm{b}_{\mathrm{AP}} \mathrm{S}=\mathrm{h}^{2} \mathrm{~S}$

Car, si $h^{2}$ est l'héritabilité du caractère on $a$ : $\mathrm{b}_{\mathrm{AP}}=\mathrm{h}^{2}$

\section{2 / L'intensité de sélection}

La différentielle de sélection $S$ s'exprime dans l'unité de mesure choisie (litre, kilogramme...) ce qui rend difficile les comparaisons. Aussi on lui préfêre souvent l'intensité de sélection i, qui s'exprime en unité d'écart-type de $P$.

$\mathrm{i}=\mathrm{S} / \sigma_{\mathrm{P}}$

Il existe par ailleurs une relation simple entre le pourcentage $p$ des animaux gardés comme reproducteurs c'est-à-dire le taux de sélection, et l'intensité de sélection i. Lorsque la distribution des valeurs du critère de sélection est normale, et lorsque la population est très grande, $\mathrm{i}=\mathrm{z} / \mathrm{p}$ où $\mathrm{z}$ est l'ordonnée du point de la courbe normale réduite qui correspond au point de troncature $\mathrm{P}_{\mathrm{t}}$ de la figure 1 .

\section{3 / Le progrès génétique}

En rapprochant $S=i \sigma_{p}$ et $R=b_{A P} S$

il vient $R=i b_{A P} \sigma_{P}$

mais $b_{A P}=\frac{\sigma_{A}^{2}}{\sigma_{P}^{2}}$ donc $R=i \frac{\sigma_{A}^{2}}{\sigma_{P}^{2}} \sigma_{P}=i \frac{\sigma_{A}}{\sigma_{P}} \sigma_{A}$

ce qui s'écrit encore, avec $h=\frac{\sigma_{A}}{\sigma_{P}}, \quad R=i h \sigma_{A}$
D'une manière plus générale, $h$, la racine carrée de l'héritabilité, est la précision $\mathrm{r}_{\mathrm{PA}}$ de la méthode d'évaluation utilisée. Lorsque la sélection se fait d'après l'indice I, on a de la même façon : $R=i r_{I A} \sigma_{A}$.

Si l'intervalle entre générations est $t$ (exprimé en année), le progrès génétique annuel $\Delta \mathrm{G}$ s'écrit :

$$
\Delta \mathrm{G}=\frac{\mathrm{ir}_{1 \mathrm{~A}} \sigma_{\mathrm{A}}}{\mathrm{t}}
$$

$\Delta \mathrm{G}$ est exprimé dans la même unité de mesure que le caractère sélectionné. L'intervalle entre générations, t, est l'âge moyen des parents à la naissance de leurs descendants qui seront soumis à la sélection pour produire la génération suivante. Dans la pratique les modalités de la sélection diffèrent dans les sexes ou le long des quatres voies de diffusion : voie père-fils (1), voie père-fille $(2)$, voie mère-fils $(3)$, voie mère-fille (4). Le progrès génétique annuel dans une forme plus générale s'écrit :

$$
\Delta G=\frac{i_{1} r_{1 A}+i_{2} r_{I A}+i_{3} r_{I A}+i_{4} r_{I A}}{t_{1}+t_{2}+t_{3}+t_{4}} \sigma_{A}
$$

les indices - 1 à 4 - désignent les quatres voies de diffusion du progrès génétique.

\section{2 / Analyse de quelques exemples}

\section{1 / Comparaison de deux intensités de sélection et de deux tailles de population}

Cet exemple est issu d'une expérience réalisée chez la souris par Hanrahan et al (1973). Ces auteurs sélectionnent sur le gain de poids post-sevrage, c'està-dire entre les âges de trois et de six semaines. Dans la population, la valeur moyenne du caractère dans le sexe mâle est d'environ $18 \mathrm{~g}$, avec un écart-type phénotypique $\sigma_{\mathrm{P}}$ de $2,49 \mathrm{~g}$ et un écart-type génétique $\sigma_{\mathrm{A}}$ de 1,35 g. A la naissance, on égalise les portées et on garde quatre mâles et quatre femelles. On sélectionne les reproducteurs intra-portée. On réalise soit une intensité de sélection maximum $(\mathrm{M})$ en retenant un reproducteur par sexe et par portée $(p=0,25)$, soit une intensité de sélection intermédiaire (I) en tirant au sort deux souriceaux par sexe et par portée puis en retenant le meilleur $(\mathrm{p}=0,50)$. On compare deux populations : la première se compose de 8 couples (8) et la seconde de 16 couples (16). On a donc quatre modalités en combinant l'intensité et la taille de la population : M8, M16, I8 et I16. On compare ces quatre populations sélectionnées à deux populations témoins, c'est-à-dire non sélectionnées et composées de 16 couples (C16-1 et C16-2). La figure 2 illustre les fluctuations des performances des souris des populations témoins. Les effets du milieu sont importants et on comprend qu'il sera difficile d'analyser une expérience de sélection en l'absence d'un témoin. La figure 3 présente la réponse à la sélection et les progrès génétiques par génération.

L'expérience a duré 14 générations ; on observe des réponses qui varient entre deux et huit grammes, soit plusieurs écart-types. Comme on l'imaginait, la réponse à la sélection augmente avec l'intensité de la sélection, et avec la taille de la population. Dans les petites populations, la variabilité génétique s'épuise plus rapidement que dans les grandes et la sélection est moins efficace. 
Figure 2. Evolution du gain de poids post-sevrage, exprimé en $g$, dans 2 souches de souris non sélectionnées.

Gain de poids post-sevrage $(\mathrm{g})$

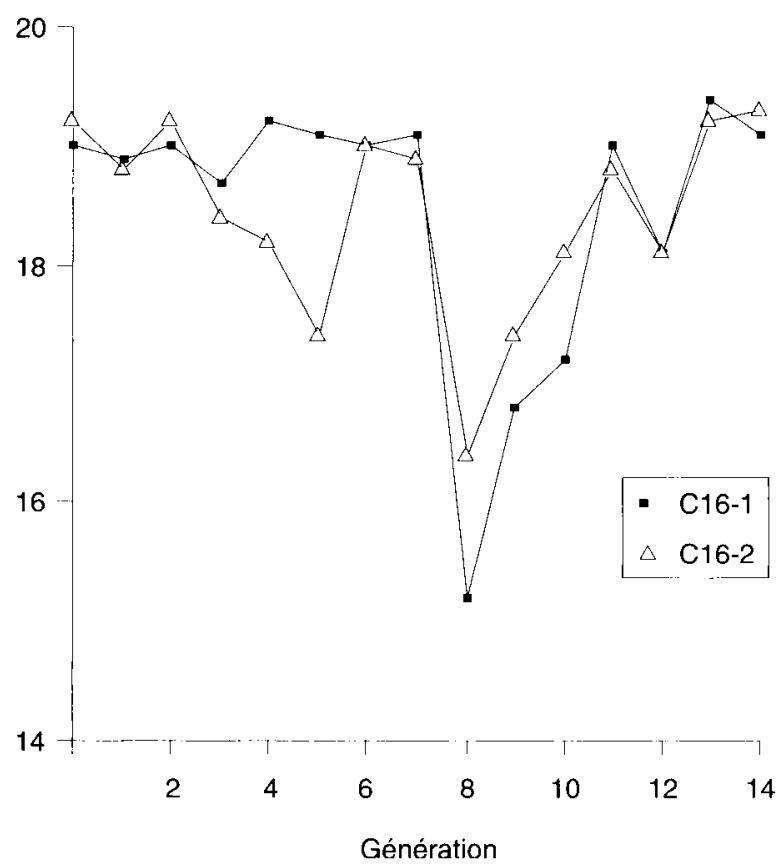

Figure 3. Evolution de la réponse à une sélection pour le gain de poids post-sevrage, exprimée en écart à deux souches témoins, dans quatre souches sélectionnées.

Gain de poids (écart à la population témoin)

(g)

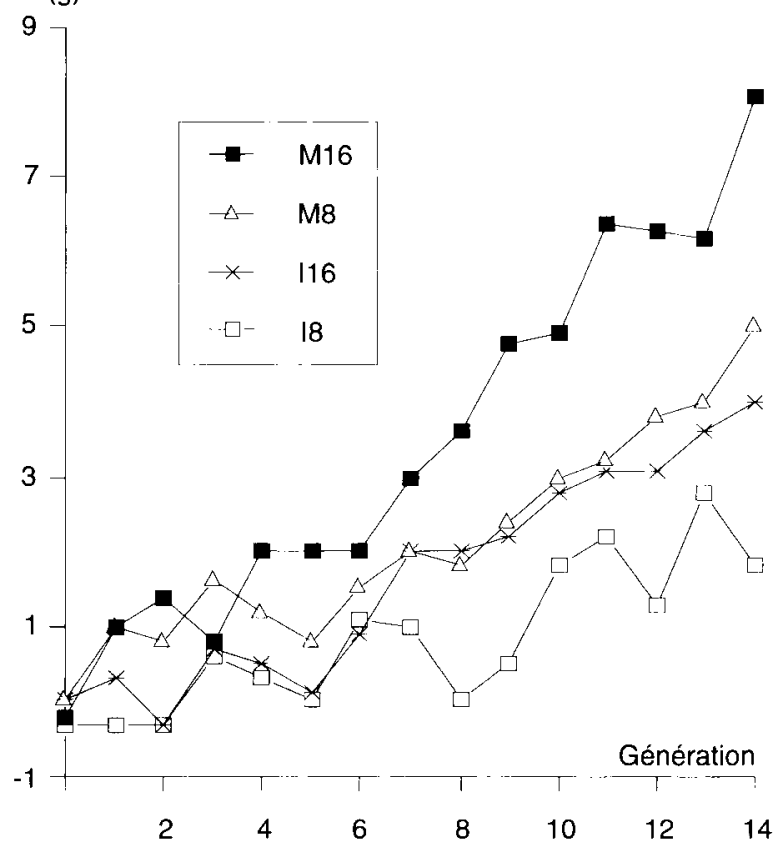

Progrès génétique par génération

\begin{tabular}{|l|c|c|c|c|}
\hline & 18 & $M 8$ & 116 & $M 16$ \\
\hline Progrès génétique $\mathrm{R}(\mathrm{g})$ & 0,15 & 0,27 & 0,31 & 0,55 \\
\hline $\mathrm{R}$ en $\%$ de $\sigma_{\mathrm{P}}$ & 6 & 11 & 12 & 22 \\
\hline $\mathrm{R}$ en $\%$ de $\sigma_{\mathrm{A}}$ & 11 & 20 & 23 & 40 \\
\hline
\end{tabular}

Nombre d'œufs en 497 jours

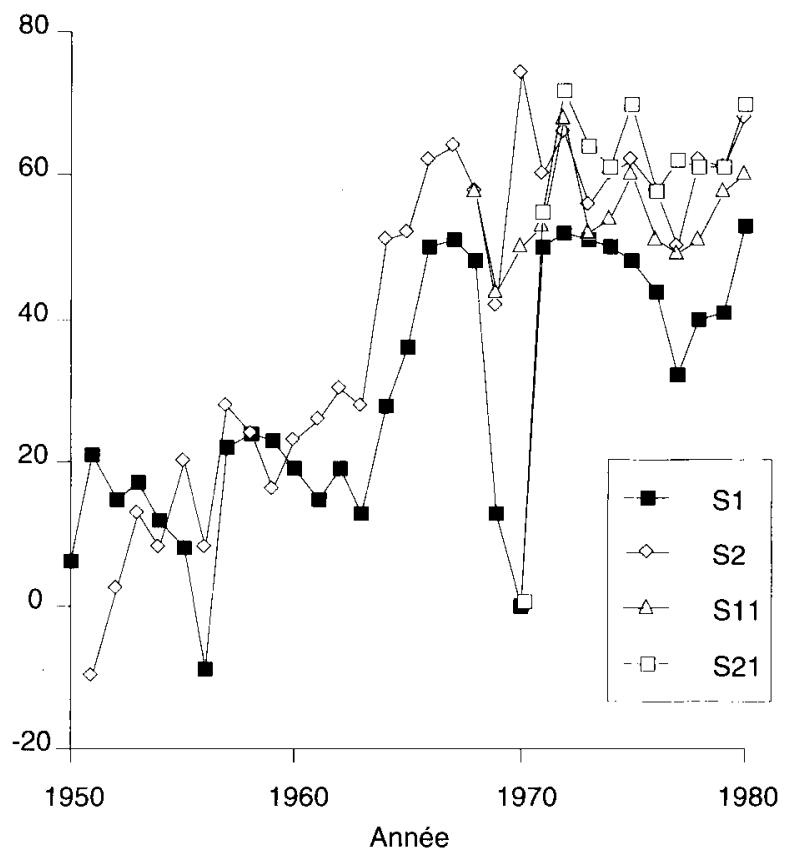

\section{2 / Comparaison entre plusieurs réplications sur une longue période de temps}

Cet exemple est issu d'une expérience réalisée chez la poule et rapportée par Fairfull et Gowe (1990). Ces auteurs de l'expérience sélectionnent sur la ponte des souches composées de 28 mâles et de 224 femelles. Le nombre d'oeufs est le critère principal, mais d'autres caractères interviennent aussi, comme par exemple le poids de l'oeuf, le poids de la poule et les caractéristiques de l'oeuf. Cette expérience a débuté en 1950 et elle s'est achevée en 1980. Jusqu'en 1966 il y avait deux souches sélectionnées et une souche témoin. Par la suite on a créé de nouvelles souches par division des souches existantes. Il y avait à la fín quatre souches sélectionnées. La figure 4 illustre l'évolution du nombre d'oeufs pondus à l'âge de 497 jours - c'està-dire après environ une année de ponte. L'effet du milieu se combine avec un effet propre à chaque réplication. D'une souche à l'autre l'effet de la sélection varie aléatoirement autour d'une tendance générale. Chez la poule il y a une génération par an. On constate donc qu'après 30 générations, la sélection est toujours efficace. La réponse moyenne à la sélection est d'environ 60 oeufs, soit $30 \%$ de la moyenne du caractère dans les souches témoins.

\section{3 / Définition des différentes modalités de sélection, et de leur effet sur les composantes du progrès génétique}

Les exemples précédents concernaient uniquement la sélection individuelle (I) ou massale, c'est-à-dire une sélection où l'on choisit les reproducteurs d'après leurs propres performances. Les performances des individus apparentés aux candidats sont des sources d'information complémentaires. On distingue classiquement trois types d'apparentés : les ascendants, les
Figure 4.

Evolution de

la réponse à

une sélection

sur le nombre

d'oeufs

pondus en

497 jours,

exprimée en

écart à une

souche (T1)

témoin, dans

quatre

souches

sélectionnées

(S1, S2, S11

et S21). 
collatéraux et les descendants, et donc trois autres modalités de sélection : la sélection sur ascendance (G), la sélection sur collatéraux (C), et la sélection sur descendance $(P)$. Le tableau 1 synthétise l'effet de chacune de ses modalités sur les trois principales composantes du progrès génétique (i : l'intensité $; r_{I A}$ : la précision ; $t$ : l'intervalle entre générations). On considère en première approximation que ces modalités n'ont pas d'effet sur la quatrième composante $\sigma_{\mathrm{A}}$. D'une manière générale, i est fixé par le sélectionneur, $\mathbf{r}_{\mathrm{IA}}$ dépend d'abord de la modalité choisie et $t$ de l'espèce sélectionnée.

On compare chaque modalité à la sélection individuelle. Un + indique que la modalité a un effet favorable sur la composante étudiée, effet qui se traduira par une augmentation du progrès génétique. Un indique un effet défavorable. Les différentes modalités sont complémentaires. La sélection sur ascendance peut s'effectuer dès la naissance, mais elle est peu précise. La sélection sur descendance est très précise, mais elle est coûteuse et tardive. Dans la pratique un plan de sélection combine ces différentes modalités.
Tableau 1. Relation entre les modalités de sélection et les composantes du progrès génétique.

\begin{tabular}{|c|c|c|c|c|}
\hline & $\mathrm{I}$ & $\mathrm{G}$ & $\mathrm{C}$ & $\mathrm{P}$ \\
\hline $\mathrm{i}$ & $=$ & + & $=$ & - \\
\hline $\mathrm{r}_{\mathrm{IA}}$ & $=$ & - & $=/+$ & ++ \\
\hline $\mathrm{t}$ & $=$ & ++ & $=$ & - \\
\hline
\end{tabular}

\section{Conclusion}

Le progrès génétique est fonction de l'intensité, de la précision et de l'intervalle entre générations ainsi que de la variabilité génétique. Les expériences de sélection confirment la possibilité de réaliser un progrès génétique.

\section{Références bibliographiques}

Bulmer M.G., 1971. The effect of selection on genetic variability. American Natur., 105, 201-211.

Fairfull R.W., Gowe R.S., 1990. Genetics of egg production in chickens. In Crawford R.D., 1990. Poultry breeding and Genetics, Elsevier, Amsterdam, 705-760.

Hanrahan J.P., Eisen E.J., Legates R., 1973. Effects of population size and selection intensity on short-term response to selection for postweaning gain in mice. Genetics, $73,513-530$.

Hill W.G., 1972. Estimation of genetic change. I. General theory and design of control populations. Anim. Breed. Abstr., 40, 1-15.

Hill W.G., 1974. Prediction and evaluation of response to selection with overlapping generations. Anim. Prod., 18, 117-139.
James J.W., 1977. Open nucleus breeding systems. Anim. Prod., 24, 287-305.

Minvielle F., 1990. Principes d'amélioration génétique des animaux domestiques. INRA, Paris. Les Presses de l'Université de Laval, Québec

Ollivier L., 1974. Optimum replacement rates in animal breeding. Anim. Prod., 19, 1-10.

Ollivier L., 1981. Eléments de génétique quantitative. Masson, Paris.

Rendel J.M., Robertson A., 1950. Estimation of genetic gain in milk yield by selection in a closed herd of dairy cattle. J. Genet., 50, 1-8. 